\title{
Electromagnetic momentum balance equation and the force density in material media
}

\author{
I. Campos ${ }^{\boxplus}$, J.L. Jiménez ${ }^{2}$ and M.A. López-Mariño ${ }^{3}$ \\ ${ }^{1}$ Departamento de Física, Facultad de Ciencias, \\ Universidad Nacional Autónoma de México, México D.F., México. \\ ${ }^{2}$ Departamento de Física, División de Ciencias Básicas e Ingeniería, \\ Universidad Autónoma Metropolitana, México D.F., México. \\ ${ }^{3}$ Departamento de Ingeniería, Tecnológico de Monterrey, \\ Campus Central de Veracruz, Córdoba, Veracruz, México.
}

Recebido em 9/9/2011; Aceito em 23/1/2012; Publicado em 20/4/2012

\begin{abstract}
We present a momentum balance equation derived directly from Maxwell's equations. This equation contains a force density, which we call Maxwell's force density, which generalizes the Lorentz force density, now including total fields rather than only external fields, and arbitrary charge and current distributions. As a test for this balance equation we derive for gases the electrostatic and magnetostatic Helmholtz force densities. This deduction will be useful for advanced undergraduates and graduate students, as well as for specialists interested in the conceptual aspects of electromagnetism.
\end{abstract}

Keywords: Maxwell's equations, Balance equation, electromagnetic force density.

Apresentamos uma equação de balanço do momento eletromagnético deduzida diretamente das equações de Maxwell. Esta equação contém uma densidade de força, por nós chamada de densidade de força de Maxwell, que generaliza aquela de Lorentz, incluindo neste caso os campos totais e não apenas os externos, bem como distribuições arbitrárias de cargas e correntes. Como teste desta equação deduzimos as forças de densidade magnetostática e eletrostática de Helmholtz para o caso de gases. Estas deduções são úteis para estudantes avançados de graduação e pós-graduação, bem como especialistas interessados em aspectos conceituais do eletromagnetismo.

Palavras-chave: equações de Maxwell, equação de balanço, densidade de força eletromagnética.

\section{Introduction}

Classical electromagnetism deals with the relation between charges and currents and electromagnetic fields in a given region of physical space that may contain material media. The fundamental equations, Maxwell's equations, give us the electromagnetic fields if the charge and current densities are given as functions of space, usually in a defined finite region, and time. These solutions constitute the inhomogeneous solutions of the equations, but there are also homogeneous solutions that are not related to the charge and currents in the region. The homogeneous plus inhomogeneous solutions must satisfy given boundary conditions and constitute the solution to a given problem.

The question of how these fields act on charges and currents is answered with the Lorentz force law

\footnotetext{
${ }^{1}$ E-mail: iecampos@prodigy.net.mx.

$$
\mathbf{F}=q\left(\mathbf{E}+\frac{\mathbf{v}}{c} \times \mathbf{B}\right)
$$

where the fields $\mathbf{E}$ and $\mathbf{B}$ are not produced by $q$ and are assumed known; these external fields are identified with homogeneous solutions, i.e., external fields [1, 2 p. 236, 572).

This force law has been considered an additional postulate to the Maxwell equations. However, in relativity it has been considered a consequence of the Lorentz transformations [1].

In the present work we show that the Lorentz law can be deduced from Maxwell's equations, but appearing in the law the total fields, external and self-fields. To this end we develop an electromagnetic momentum balance equation, and show that in the static case this balance equation contains, for gases, Helmholtz's force 
density. This balance equation permits also analyze the concept of test charges.

\section{The momentum balance}

Now we proceed to deduce the momentum balance equation with the method used to deduce the energy balance in a previous work [3]. The central point of the method is to use only Maxwell's equations and avoid the use of Lorentz's law, since the fields appearing in the law are only the external fields, i.e., not produced by $\rho$ and $\mathbf{J}[1,2$ p. 236, 572]. Self-interaction appears as the interaction of infinitesimal charges acting on other infinitesimal charges of the charge distribution, as Lorentz [4] (see also Refs. [2, 5, 6]) does in his treatment of radiation reaction.

The derivation of the momentum balance equation we present in this work is straightforward and, since we do not use Lorentz's law the fields involved are the total fields. Thus the stress-energy tensor, which integrated over a closed surface that contains a charge-current distribution gives the electromagnetic force on this distribution, will include the fields associated to the charge and current distributions outside the closed surface, as well as the self fields of the charges and currents inside, i.e., the total fields. Then the present deduction differs of the one usually given in standard texts, like Jackson's $[2,7]$.

We start with the macroscopic Maxwell equations [3]

$$
\begin{array}{r}
\nabla \cdot \mathbf{D}=4 \pi \rho, \\
\nabla \cdot \mathbf{B}=0, \\
\nabla \times \mathbf{E}=-\frac{1}{c} \frac{\partial \mathbf{B}}{\partial t}, \\
\nabla \times \mathbf{H}=\frac{4 \pi}{c} \mathbf{J}+\frac{1}{c} \frac{\partial \mathbf{D}}{\partial t} .
\end{array}
$$

Now we multiply Eq. (2) by E, Eq. (3) by $\mathbf{H}$, and multiply vectorially on the left, Eq. (4) by D, and Eq. (5) by B. Adding these results and rearranging terms leads to the expression

$$
\begin{array}{r}
\frac{1}{4 \pi}[\mathbf{E}(\nabla \cdot \mathbf{D})-\mathbf{D} \times(\nabla \times \mathbf{E})+\mathbf{H}(\nabla \cdot \mathbf{B})- \\
\mathbf{B} \times(\nabla \times \mathbf{H})]-\frac{1}{4 \pi c} \frac{\partial}{\partial t}(\mathbf{D} \times \mathbf{B})=\rho \mathbf{E}+\frac{\mathbf{J}}{c} \times \mathbf{B} .
\end{array}
$$

Since we want to express this equation as a balance equation it is necessary to rewrite the first four terms on the left, and it is convenient to define

$$
\mathbf{E}(\nabla \cdot \mathbf{D})-\mathbf{D} \times(\nabla \times \mathbf{E})=\mathbf{T}_{1}
$$

and

$$
\mathbf{H}(\nabla \cdot \mathbf{B})-\mathbf{B} \times(\nabla \times \mathbf{H})=\mathbf{T}_{2} .
$$

It is important to note that these expressions exhibit the symmetry between the fields $\mathbf{E}$ and $\mathbf{H}$, and between $\mathbf{D}$ and $\mathbf{B}$ inherent in the macroscopic Maxwell equations. The fields $\mathbf{E}$ and $\mathbf{H}$ appear in line integrals or in rotationals, while the fields $\mathbf{D}$ and $\mathbf{B}$ appear in surface integrals or in divergences. These features hold trough the entire deduction, then it is enough to show how to rewrite $\mathbf{T}_{1}$ since $\mathbf{T}_{2}$ can be rewritten in the same way.

We need the following vector identities,

$$
\begin{gathered}
\nabla \cdot(\mathbf{u v})=\mathbf{v}(\nabla \cdot \mathbf{u})+(\mathbf{u} \cdot \nabla) \mathbf{v} \\
\nabla(\mathbf{u} \cdot \mathbf{v})=(\mathbf{u} \cdot \nabla) \mathbf{v}+(\mathbf{v} \cdot \nabla) \\
\mathbf{u}+\mathbf{u} \times(\nabla \times \mathbf{v})+\mathbf{v} \times(\nabla \times \mathbf{u}) \\
\mathbf{u} \times(\nabla \times \mathbf{v})=(\nabla \mathbf{v}) \cdot \mathbf{u}-(\mathbf{u} \cdot \nabla) \mathbf{v}
\end{gathered}
$$

Equation (9) refers to a tensor or dyadic product.

With these identities we avoid Jackson's [2, 7] procedure, which uses Maxwell's equations in terms of components. If we use Eqs. (10) and (11) with $\mathbf{u}=\mathbf{D}$ and $\mathbf{v}=\mathbf{E}$, we have

$$
\begin{aligned}
& \mathbf{T}_{1}=\mathbf{E}(\nabla \cdot \mathbf{D})-\frac{1}{2} \mathbf{D} \times(\nabla \times \mathbf{E})- \\
& \frac{1}{2} \mathbf{D} \times(\nabla \times \mathbf{E})=\mathbf{E}(\nabla \cdot \mathbf{D})-\frac{1}{2} \nabla(\mathbf{D} \cdot \mathbf{E})+ \\
& \frac{1}{2}[(\mathbf{D} \cdot \nabla) \mathbf{E}+(\mathbf{E} \cdot \nabla) \mathbf{D}+\mathbf{E} \times(\nabla \times \mathbf{D})]- \\
& \frac{1}{2}[(\nabla \mathbf{E}) \cdot \mathbf{D}-(\mathbf{D} \cdot \nabla) \mathbf{E}] .
\end{aligned}
$$

The trick of expressing $\mathbf{D} \times(\nabla \times \mathbf{E})$ as

$$
\frac{1}{2}(\mathbf{D} \times(\nabla \times \mathbf{E}))+\frac{1}{2}(\mathbf{D} \times(\nabla \times \mathbf{E}))
$$

is useful since this term appears both, in Eq. (10) and Eq.(11), then we assign an equal contribution to each equation.

Now, in order to avoid the term $\mathbf{E} \times(\nabla \times \mathbf{D})$ we use the identity (11) with $\mathbf{u}=\mathbf{E}$, and $\mathbf{v}=\mathbf{D}$. This permits us regrouping the terms obtaining

$$
\begin{aligned}
& \mathbf{T}_{1}=\mathbf{E}(\nabla \cdot \mathbf{D})-\frac{1}{2} \nabla(\mathbf{D} \cdot \mathbf{E})+\frac{1}{2}(\mathbf{D} \cdot \nabla) \mathbf{E}+ \\
& \frac{1}{2}(\nabla \mathbf{D}) \cdot \mathbf{E}-\frac{1}{2}(\nabla \mathbf{E}) \cdot \mathbf{D}+\frac{1}{2}(\mathbf{D} \cdot \nabla) \mathbf{E}= \\
& \mathbf{E}(\nabla \cdot \mathbf{D})+(\mathbf{D} \cdot \nabla) \mathbf{E}-\frac{1}{2} \nabla(\mathbf{D} \cdot \mathbf{E})+ \\
& \frac{1}{2}[(\nabla \mathbf{D}) \cdot \mathbf{E}-(\nabla \mathbf{E}) \cdot \mathbf{D}] .
\end{aligned}
$$


Finally we use Eq. (9), which permits us regroup some terms and obtain the result we were looking for

$$
\begin{aligned}
& \mathbf{T}_{1}=\nabla \cdot\left(\mathbf{D E}-\frac{1}{2} \mathbf{D} \cdot \mathbf{E I}\right)+ \\
& \frac{1}{2}[(\nabla \mathbf{D}) \cdot \mathbf{E}-(\nabla \mathbf{E}) \cdot \mathbf{D}],
\end{aligned}
$$

Proceeding analogously with the magnetic fields, we obtain

$$
\begin{aligned}
& \mathbf{T}_{2}=\nabla \cdot\left(\mathbf{B H}-\frac{1}{2} \mathbf{B} \cdot \mathbf{H I}\right)+ \\
& \frac{1}{2}[(\nabla \mathbf{B}) \cdot \mathbf{H}-(\nabla \mathbf{H}) \cdot \mathbf{B}] .
\end{aligned}
$$

With the results obtained for $\mathbf{T}_{1}$ and $\mathbf{T}_{2}$ in Eqs. (14) and (15) we can transform Eq. (6) into the expression

$$
\begin{aligned}
& \frac{1}{4 \pi} \nabla \cdot\left\{\mathbf{D E}+\mathbf{B H}-\frac{1}{2}(\mathbf{D} \cdot \mathbf{E}+\mathbf{B} \cdot \mathbf{H}) \mathbf{I}\right\}- \\
& \frac{1}{4 \pi c} \frac{\partial}{\partial t}(\mathbf{D} \times \mathbf{B})=\rho \mathbf{E}+\frac{\mathbf{J}}{c} \times \mathbf{B}+ \\
& \frac{1}{8 \pi}\{(\nabla \mathbf{E}) \cdot \mathbf{D}-(\nabla \mathbf{D}) \cdot \mathbf{E}+(\nabla \mathbf{H}) \cdot \mathbf{B}- \\
& (\nabla \mathbf{B}) \cdot \mathbf{H}\},
\end{aligned}
$$

which is the momentum balance equation we were looking for. It is convenient to write it as

$$
\nabla \cdot \mathbf{T}-\frac{\partial \mathbf{g}_{M}}{\partial t}=\mathbf{f}_{\text {Maxwell }}
$$

where, evidently,

$$
\mathbf{g}_{M}=\frac{1}{4 \pi c} \mathbf{D} \times \mathbf{B}
$$

which is the momentum density of the electromagnetic field proposed by Minkowski. The stress tensor in terms of dyadics is

$$
\mathbf{T}=\frac{1}{4 \pi}\left[\mathbf{D E}+\mathbf{B H}-\frac{1}{2}(\mathbf{D} \cdot \mathbf{E}+\mathbf{B} \cdot \mathbf{H}) \mathbf{I}\right],
$$

and the force density happens to be

$$
\begin{aligned}
& \mathbf{f}_{\text {Maxwell }}=\rho \mathbf{E}+\frac{\mathbf{J}}{c} \times \mathbf{B}+\frac{1}{8 \pi}\{(\nabla \mathbf{E}) \cdot \mathbf{D}- \\
& (\nabla \mathbf{D}) \cdot \mathbf{E}+(\nabla \mathbf{H}) \cdot \mathbf{B}-(\nabla \mathbf{B}) \cdot \mathbf{H}\},
\end{aligned}
$$

which we call the Maxwell force density. This force density includes, besides the Lorentz force with total fields, other force densities, like Helmholtz's, for gases.
On the other hand, it can be shown that in static conditions a finite point charge is acted on only by external fields. That the forces on charges and currents are transmitted by stresses associated to the electromagnetic fields is Faraday's original idea, later formalized by Maxwell. This view can be illustrated by some examples in electrostatics. We have so that Coulomb's force law can be derived by surface integration of the stress tensor [5, p. 106-107 and p. 116, problem 1), which contains the field of the charge inside the closed surface, the proper field, and the field asociated to charges outside the closed surfaces i.e. the external field thus the total field is involved. Another example is given in Konopinski's textbook [8, p. 164-166], where a finite point charge is in an initially constant field. Again the stress tensor involves the total fields, though by symmetry the self field does not exert a force on a point or spherically symmetric charge, and the usual Lorentz force results. Thus, in electrostatics finite point charges can play the role of test charges. Thus in electrostatics the concept of test charge as an infinitesimal point charge is unnecessary. Let us note that non electromagnetic forces are involved in the mechanical equilibrium of a system of finite point charges. However for charges in motion this is not the case due to the effects of retardation.

\section{The momentum balance equation and the Helmholtz force density}

Now we show what these general results obtained from Maxwell's equations imply in particular cases. Let us consider the electrostatic case. In these static conditions the momentum balance equation reduces to

$$
\begin{aligned}
& \nabla \cdot\left\{\frac{1}{4 \pi}\left[\mathbf{E D}-\frac{1}{2}(\mathbf{D} \cdot \mathbf{E I})\right]\right\}=\rho \mathbf{E}+ \\
& \frac{1}{8 \pi}\{(\nabla \mathbf{E}) \cdot \mathbf{D}-(\nabla \mathbf{D}) \cdot \mathbf{E}\} .
\end{aligned}
$$

Now, if we use the constitutive relation

$$
\mathbf{D}=\varepsilon \mathbf{E}
$$

in the right hand side of Eq. (21), while in the scalar product in the left term we use the constitutive relation now expressed as

$$
\mathbf{D}=\mathbf{E}+4 \pi \mathbf{P},
$$

we obtain immediatly

$$
\begin{aligned}
& \nabla \cdot\left\{\frac{1}{4 \pi}\left[\mathbf{D E}-\frac{1}{2} \mathbf{E}^{2} \mathbf{I}\right]-\frac{1}{2}(\mathbf{P} \cdot \mathbf{E}) \mathbf{I}\right\}= \\
& \rho \mathbf{E}-\frac{1}{8 \pi} \mathbf{E}^{2} \nabla \varepsilon
\end{aligned}
$$

or 


$$
\begin{aligned}
& \nabla \cdot\left\{\frac{1}{4 \pi}\left[\mathbf{D E}-\frac{1}{2} \mathbf{E}^{2} \mathbf{I}\right]\right\}=\rho \mathbf{E}-\frac{1}{8 \pi} \mathbf{E}^{2} \nabla \varepsilon+ \\
& \nabla\left(\frac{1}{2} \mathbf{P} \cdot \mathbf{E}\right) .
\end{aligned}
$$

It is also satisfied that, for linear media,

$$
\mathbf{P}=\chi \mathbf{E},
$$

then, Eq. (25) can be written as

$$
\begin{aligned}
& \nabla \cdot\left\{\frac{1}{4 \pi}\left[\mathbf{E D}-\frac{1}{2} E^{2} \mathbf{I}\right]\right\}=\rho \mathbf{E}- \\
& \frac{1}{8 \pi} E^{2} \nabla \varepsilon+\nabla\left(\frac{1}{2} \chi E^{2}\right) .
\end{aligned}
$$

Furthermore, if we consider a fluid for which

$$
\chi=\frac{1}{4 \pi} \rho_{m} \frac{\partial \varepsilon}{\partial \rho_{m}}
$$

holds [7], then we can write Eq. (27) in the form

$$
\begin{aligned}
& \nabla \cdot\left\{\frac{1}{4 \pi}\left[\mathbf{D E}-\frac{1}{2} E^{2} \mathbf{I}\right]\right\}=\rho \mathbf{E}- \\
& \frac{1}{8 \pi} E^{2} \nabla \varepsilon+\frac{1}{8 \pi} \nabla\left(\rho_{m} \frac{\partial \varepsilon}{\partial \rho_{m}} E^{2}\right),
\end{aligned}
$$

which is the balance equation Eq. (24), but in the right hand side appears the force density obtained by Helmholtz.

Given the symmetry with which the fields $\mathbf{E}$ and $\mathbf{H}$ appear in Eq. (16), it is immediate to see that for the magnetic field $\mathbf{H}$ the following balance equation results,

$$
\begin{aligned}
& \nabla \cdot\left\{\frac{1}{4 \pi}\left[\mathbf{B H}-\frac{1}{2} H^{2} \mathbf{I}\right]\right\}=\frac{1}{c} \mathbf{J} \times \mathbf{B}- \\
& \frac{1}{8 \pi} H^{2} \nabla \mu+\frac{1}{8 \pi} \nabla\left(\rho_{m} \frac{\partial \mu}{\partial \rho_{m}} H^{2}\right) .
\end{aligned}
$$

With these results we obtain that the original balance equation can be written as

$$
\begin{aligned}
& \nabla \cdot\left\{\frac{1}{4 \pi}\left[\mathbf{D E}+\mathbf{B H}-\frac{1}{2}\left(E^{2}+H^{2}\right) \mathbf{I}\right]\right\}= \\
& =\rho \mathbf{E}+\frac{1}{c} \mathbf{J} \times \mathbf{B}-\frac{1}{8 \pi}\left(E^{2} \nabla \varepsilon+H^{2} \nabla \mu\right)+ \\
& \frac{1}{8 \pi} \nabla\left(\rho_{m} \frac{\partial \varepsilon}{\partial \rho_{m}} E^{2}+\rho_{m} \frac{\partial \mu}{\partial \rho_{m}} H^{2}\right) .
\end{aligned}
$$

These results are not free of criticisms, and authors like Robinson [9] consider this kind of deductions mere identities, and so he considers that the "correct" Helmholtz force density is the one deduced from Maxwell's equations and fluid mechanics [5, 10]. But we can see that both expressions, Eqs. (29) and (30) are contained in what we call Maxwell's force density. For a deeper discussion see Jiménez et al. [12].

Other authors, like Panofsky and Phillips [5] and Jackson [2], try to incorporate the Helmholtz force density in a momentum balance equation of the type

$$
\nabla \cdot \mathbf{T}^{*}-\frac{\partial \mathbf{g}_{A}}{\partial t}=\mathbf{f}_{H e l m}
$$

by rewritting the stress tensor, as $\mathbf{T}^{*}=\mathbf{T}_{\text {ele }}^{*}+\mathbf{T}_{\text {mag }}^{*}$, where

$$
\mathbf{T}_{\text {ele }}^{*}=\frac{1}{4 \pi}\left[\mathbf{D E}-\frac{\mathbf{I}}{2}(\mathbf{E} \cdot \mathbf{D})\left(1-\frac{\rho_{m}}{\varepsilon} \frac{\partial \varepsilon}{\partial \rho_{m}}\right)\right],
$$

and

$$
\mathbf{T}_{m a g}^{*}=\frac{1}{4 \pi}\left[\mathbf{B H}-\frac{\mathbf{I}}{2}(\mathbf{B} \cdot \mathbf{H})\left(1-\frac{\rho_{m}}{\mu} \frac{\partial \mu}{\partial \rho_{m}}\right)\right] .
$$

We can see from the present deduction that this is not necessary, since the electromagnetic tensor $\mathbf{T}$ already contains the pertinent information for gases or fluids obeying Eq. (28), and it is not necessary to rewrite it. This is an important point to note, and therefore the criticism by Robinson [9] is not well founded, contrasting it with the generality of the present approach.

\section{Conclusions}

We have shown in the present work that, by using the vectorial identities, Eqs. (9)-(11), it is possible to obtain directly from Maxwell's equations the momentum balance equation, identifying in it a force density that we have called Maxwell's force density. Robinson's criticism [9] that this type of deduction is a mere identity does not constitute a demerit; otherwise we would have to blame also the continuity equation of charge and current, since it also results from an identity derived from Maxwell's equations. It is also clear from this deduction that the usual Lorentz force is an approximation for very small charges and corresponding currents, which permits to neglect the self fields. Therefore the generalized Lorentz force, including total fields, is implicit in Maxwell's equations. The inclusion of total fields is essential to the study of radiation reaction. On the other hand such a general result like this must be tested with particular models and experiments. In this sense is remarkable that the Helmholtz force density for fluids is contained, as a particular case, in the Maxwell balance equation. This is a relevant result not appearing in the 
literature. A final point, relevant for teaching, is the use of vector identities, not all familiar to students that permit a straightforward deduction, avoiding in this way the tricky deductions with components that obscure the deduction and make it repellent to the reader.

\section{References}

[1] M Bunge, Foundations of Physics (Springer, Berlin, 1967).

[2] J.D. Jackson, Classical Electrodynamics (Wiley, New York, 1975), $2^{\text {nd }}$ ed.

[3] I. Campos and J.L. Jiménez, Eur. J. Phys. 13, 117 (1992).

[4] H.A. Lorentz, The Theory of Electrons and Its Applications to Phenomena of Light and Radiant Heat (Dover, New York, 1952), $2^{\text {nd }}$ ed. In 1916 Teubner published the second edition of Lectures delivered by the author at Columbia University, New York, in 1906.
[5] W.K.H. Panofsky and M. Phillips, Classical Electricity and Magnetism (Addison-Wesley Publishing Company Massachussetts, 1962), $2^{\text {nd }}$ ed.

[6] J. Vanderlinde, Classical Electromagnetic Theory (Springer-Verlag, New York, 2004), $2^{\text {nd }}$ ed.

[7] J.D. Jackson, Classical Electrodynamics (Wiley, New York, 1999), $3^{\text {rd }}$ ed.

[8] E.J. Konopinski, Electromagnetic Fields and Relativistic Particles (McGraw-Hill Book Company, New York, 1981).

[9] F.N.H. Robinson, Phys. Report. C 16, 313 (1975).

[10] L.D. Landau, E.M. Lifshitz and L.P. Pitaevski Electrodynamics of Continuous Media (Pergamon Press, Oxford,1984).

[11] J.A. Stratton, Electromagnetic Theory (McGraw-Hill, New York, 1941).

[12] J.L. Jiménez, I. Campos, M.A. López-Mariño A new perspective on the Abraham-Minkowski controversy, Eur. Phys. J. - Plus 12650 (2011). 